\title{
Acinetobacter Baumannii Native Valve Infective Endocarditis: A Case Report
}

\author{
Ismahane Lahmidi ${ }^{1}$, Darar Charmake III ${ }^{2}$, Noha Elouafi ${ }^{2}$, Zakaria Bazid ${ }^{2}$ \\ 1. Cardiology, Mohammed I University/Mohammed VI University Hospital, Epidemiological Laboratory of Clinical \\ Research and Public Health, Oujda, MAR 2. Cardiology, Mohammed I University/Mohammed VI University Hospital, \\ Oujda, MAR
}

Corresponding author: Ismahane Lahmidi, lahmidiismahane@gmail.com

\begin{abstract}
Infective endocarditis caused by Acinetobacter (A.) baumannii is a rare but severe complication that affects seriously ill, hospitalized patients undergoing invasive procedures. It is associated with an increased mortality rate than that of endocarditis due to the HACEK group (Haemophilus species, Aggregatibacter actinomycetemcomitans, Cardiobacterium hominis, Eikenella corrodens, Kingella kingae) gram-negative bacteria. We report a case of a 54-year-old woman who was diagnosed with infective endocarditis caused by A. baumannii three days following her admission to the intensive unit care (ICU). The diagnosis was made on the basis of repeated blood cultures and transthoracic echocardiography, which revealed mobile vegetation attached to the mitral valve. In spite of aggressive therapeutic regimens, outcomes were poor and the patient died. This report underlines the severe nature of A. baumannii infections, which are still associated with a prolonged hospital stay, and increased morbidity, mortality, and medical costs.
\end{abstract}

Categories: Cardiology, Emergency Medicine, Infectious Disease

Keywords: acinetobacter baumannii, infective endocarditis, nosocomial infections

\section{Introduction}

Infective endocarditis (IE) is a life-threatening infection involving the endocardial surface of the heart. Although uncommon, IE has a high mortality rate, even with appropriate antibiotic therapy and surgical intervention. Gram-positive cocci, mostly Staphylococcus aureus and Streptococci, are the main causes of infective endocarditis. Gram-negative bacteria and fungi are not frequent pathogens of infective endocarditis [1-2]. Acinetobacter (A.) species are ubiquitous gram-negative coccobacilli and usually cause nosocomial infections, principally ventilator-associated pneumonia and catheter-associated bacteremia, as well as soft tissue and urinary tract infections. Community-acquired infections by Acinetobacter baumannii are increasingly reported [3]. A rare case of native mitral valve endocarditis secondary to A. baumannii is described in the present report.

Review began 10/20/2020 Review ended 11/06/2020 Published 11/17/2020

(c) Copyright 2020

Lahmidi et al. This is an open access article distributed under the terms of the Creative Commons Attribution License CC-BY 4.0., which permits unrestricted use, distribution, and reproduction in any medium, provided the original author and source are credited.

\section{Case Presentation}

A 54-year-old woman presented with a month-long history of painful necrotic ulcer in the left second toe, resistant to conventional treatment. She developed severe sepsis requiring admission to the intensive care unit. On her third day of hospitalization. she developed a high fever of $39.5^{\circ} \mathrm{C}$. A new mitral systolic murmur (3/6 intensity) was noted on auscultation. Because of the high suspicion of infective endocarditis, transthoracic echocardiography (TTE) was carried out, which showed mobile vegetation measuring $0.2 \times 0.8$ $\mathrm{cm}$ attached to the mitral valve (Figure 1 ). A diagnosis of native mitral valve infective endocarditis was established according to the Duke criteria [4]. The patient subsequently was transferred to the cardiac care unit after hemodynamic stabilization. Upon arrival in our unit (cardiac care unit), the patient's clinical examination revealed a temperature of $39.8^{\circ} \mathrm{C}$, blood pressure (BP) of $110 / 60 \mathrm{mmHg}$, sinus tachycardia with a pulse of $100 / \mathrm{min}$, and respiratory rate of 20 /minute, with an oxygen saturation of $98 \%$ on room air. Cardiovascular examination revealed no signs of heart failure, a mitral systolic murmur was heard, and no peripheral stigmata of infective endocarditis were observed. There was a black ischemic ulcer on the dorsum of the left second toe, with oedema (Figure 2). Foul-smelling purulent drainage was noted on her toe ulcer. A dermatology consult was requested for skin ulcer biopsy and culture, a skin punch biopsy of her left second toe ulcer was obtained. Cultures of these skin biopsies grew Staphylococcus aureus and Candida parapsilosis but failed to isolate Acinetobacter baumannii. Histopathology of his skin biopsy reported leukocytoclastic vasculitis. Three blood cultures were carried out at an interval of an hour, as she had a high temperature $\left(40^{\circ} \mathrm{C}\right)$; another blood sample was also collected. Two sets of blood cultures yielded Acinetobacter baumannii. The antibiotic susceptibilities of the A. baumannii isolated from our patient are summarized in Table 1. 


\section{Cureus}

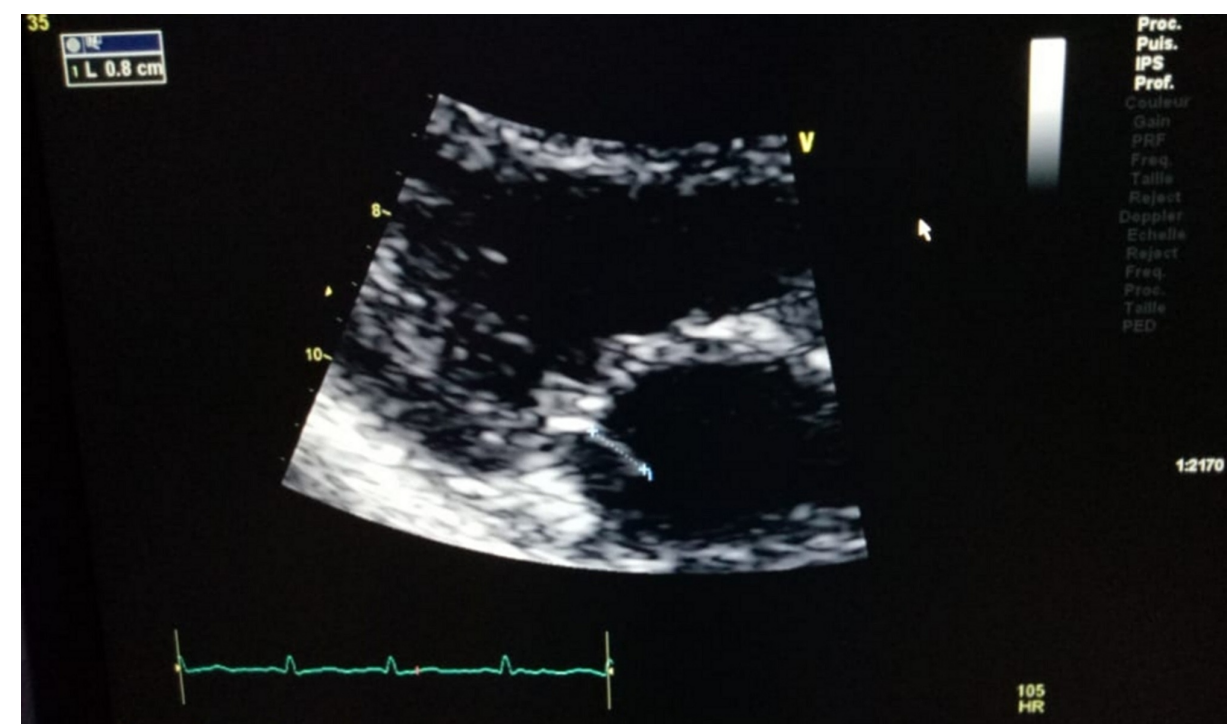

FIGURE 1: Transthoracic echocardiography showed $0.8 \times 0.2 \mathrm{~cm}$ vegetation on the patient's mitral valve in the parasternal long-axis view 


\section{Cureus}

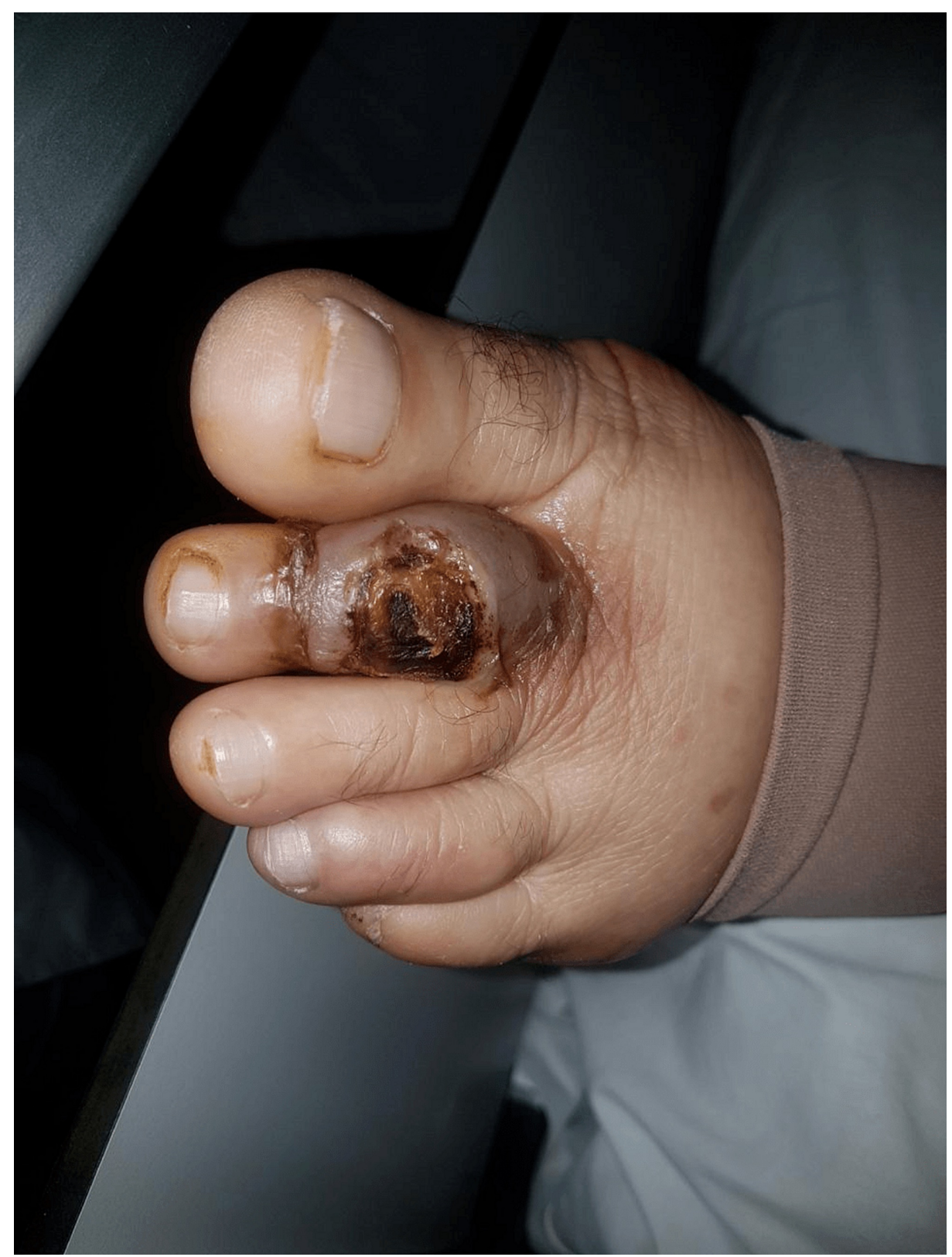

FIGURE 2: Necrotic ulcer of the patient's second left toe 


\section{Cureus}

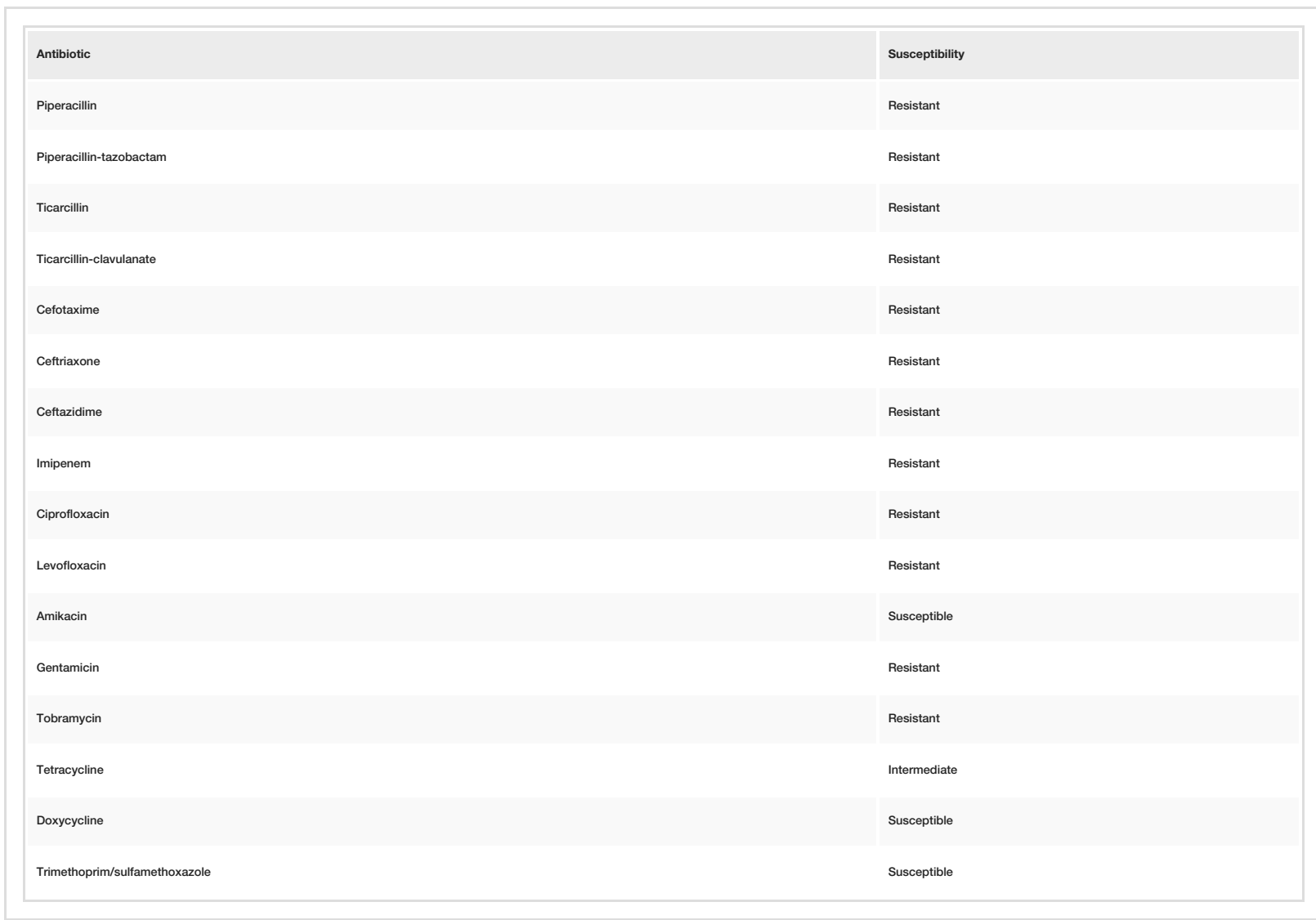

TABLE 1: Antibiotic susceptibility profile of Acinetobacter baumannii isolated from our patient

Laboratory test results revealed an elevated C-reactive protein (CRP) (100 mg/L) and erythrocyte sedimentation rate (ESR) $(110 \mathrm{~mm} /$ hour). We also noted leukocytosis (white blood cell count 22,000 cells $/ \mathrm{mm}^{3}, 80 \%$ neutrophils) and normochromic normocytic anaemia. Liver function, serum creatinine levels, and the extracted urine sample were normal. Initially, vancomycin, gentamycin, and fluconazole were started but intermittent fever and chills persisted. After blood culture results, polymyxin $\mathrm{E}$ was set for 45 days without clinical improvement. Abdominopelvic computed tomography (CT) did not show any abnormalities. Antibiotics were shifted to tigecycline, and early surgical intervention was considered. However, the patient was medically not fit for cardiac surgery with multi-organ failure, and she subsequently died.

\section{Discussion}

A. baumannii is a ubiquitous bacterial pathogen that is largely implicated in nosocomial infections. It is a pleomorphic, aerobic gram-negative bacillus, non-motile, and widely distributed in the environment. Its ability to survive even in hostile conditions allows it a wide circulation in the hospital setting. It is often spread to patients from workers' hands or healthcare devices [5]. There are many risk factors associated with Acinetobacter spp. bacteremia-like invasive procedures, mechanical ventilation, and surgery [6]. Among 17 cases of A. baumannii endocarditis reviewed [7], the inciting events were dental work, intravenous drug abuse, septic abortion, stab wounds, burns, intravenous catheters, and open-heart surgery. None of these events was noted in our patient except for the hemodialysis catheter in his right internal jugular vein. Most likely, in our patient, the infection is secondary to invasive procedures (intravascular catheter). We postulated also that the skin lesions (left second toe ulcer) could be the likely nidus of the infection.

The most frequent sites of infection are urinary and respiratory tracts, bloodstream, and surgical sites. A few cases of A. baumannii endocarditis have previously been reported. Prosthetic valves were involved in the majority of these cases [8-9].

Native valve endocarditis due to A. baumannii is a life-threatening infection, with a worse prognosis than the prosthetic valve form, perhaps because of the poor index of suspicion and the delayed initiation of treatment. Among the described patients in the literature, $16.6 \%$ of patients with prosthetic valve endocarditis died vs. $33 \%$ of patients with native valve endocarditis [7].

Echocardiography is essential for diagnosis. It should be performed in all patients with a moderate or high 
suspicion of infective endocarditis to confirm the diagnosis and guide the timely institution of appropriate therapy, including the need for surgical intervention. It also helps in determining prognosis [10]. Transesophageal echocardiography (TEE) is much more sensitive than transthoracic echocardiography (TTE), with increased diagnostic accuracy. In our case, the diagnosis was easily made without resorting to TEE.

It is evident that the outcome of patients with A.baumannii infective endocarditis is poor due to its growing resistance to antimicrobials. Imipenem is usually the first-line antibiotic against infections caused by A. baumannii [7,11], Empirical broad-spectrum therapy (e.g., imipenem/cilastatin) for Acinetobacter infections should be considered in any patient with infective endocarditis when gram-negative coccobacilli are isolated from blood cultures [7]. The present isolate in our patient was resistant to imipenem.

No surgical intervention was reported in a study of native-valve endocarditis due to Acinetobacter species [7], and a survival rate of $65 \%$ was reported. Our patient met indications for surgical treatment [12], but she was at high risk considering her poor general condition.

\section{Conclusions}

This report concluded that native valve infective endocarditis caused by A. baumannii is rare. The relevance of this case is centred on the fact that infective endocarditis due to the dreaded A. baumannii is not a common cause but a possible cause of death in patients who have stayed in the intensive care unit; hence, the necessity of nosocomial infection control and prevention measures.

\section{Additional Information \\ Disclosures}

Human subjects: Consent was obtained by all participants in this study. Conflicts of interest: In compliance with the ICMJE uniform disclosure form, all authors declare the following: Payment/services info: All authors have declared that no financial support was received from any organization for the submitted work. Financial relationships: All authors have declared that they have no financial relationships at present or within the previous three years with any organizations that might have an interest in the submitted work. Other relationships: All authors have declared that there are no other relationships or activities that could appear to have influenced the submitted work.

\section{References}

1. Mackie AS, Liu W, Savu A, Marelli AJ, Kaul P: Infective endocarditis hospitalizations before and after the 2007 American Heart Association Prophylaxis Guidelines. Can J Cardiol. 2016, 32:942-948. 10.1016/j.cjca.2015.09.021

2. Durante-Mangoni E, Andini R, Agrusta F, Iossa D, Mattucci I, Bernardo M, Utili R: Infective endocarditis due to multidrug resistant gram-negative bacilli: single centre experience over 5 years. Eur J Intern Med. 2014, 25:657-661. 10.1016/j.ejim.2014.05.015

3. Wong D, Nielsen TB, Bonomo RA, Pantapalangkoor P, Luna B, Spellberga B: Clinical and pathophysiological overview of acinetobacter infections: a century of challenges. Clin Microbiol Rev. 2017, 30:409-447. 10.1128/CMR.00058-16

4. Li JS, Sexton DJ, Mick N, et al.: Proposed modifications to the Duke criteria for the diagnosis of infective endocarditis. Clin Infect Dis. 2000, 30:633-8. 10.1086/313753

5. McDonald LC, Banerjee SN, Jarvis WR, Nosocomial Infections Surveillance System: Seasonal variation of Acinetobacter infections: 1987-1996. Clin Infect Dis. 1999, 29:1133-1137. 10.1086/313441

6. Choi S-H, Choo EJ, Kwak YG, et al.: Clinical characteristics and outcomes of bacteremia caused by Acinetobacter species other than A. baumannii: comparison with A. baumannii bacteremia. J Infect Chemother. 2006, 12:380-386. 10.1007/s10156-006-0488-1

7. Gradon JD, Chapnick EK, Lutwick LI: Infective endocarditis of a native valve due to Acinetobacter: case report and review. 1992, 14:1145-1148. 10.1093/clinids/14.5.1145

8. Falagas ME, Kopterides P: Risk factors for the isolation of multi-drug-resistant Acinetobacter baumannii and Pseudomonas aeruginosa: a systematic review of the literature. J Hosp Infect. 2006, 64:7-15. 10.1016/j.jhin.2006.04.015

9. Gaynes R, Edwards JR, National Nosocomial Infections Surveillance System: Overview of nosocomial infections caused by gram-negative bacilli. Clin Infect Dis. 2005, 41:848-854. 10.1086/432803

10. Habib G, Badano L, Tribouilloy C, et al.: Recommendations for the practice of echocardiography in infective endocarditis. Eur Heart J Cardiovasc Imaging. 2010, 11:202-219. 10.1093/ejechocard/jeq004

11. Almasaudi SB: Acinetobacter spp. as nosocomial pathogens: epidemiology and resistance features . Saudi J Biol Sci. 2018, 25: 586-596. 10.1016/j.sjbs.2016.02.009

12. Gouëllo JP, Asfar P, Brenet O, Kouatchet A, Berthelot G, Alquier P: Nosocomial endocarditis in the intensive care unit: an analysis of 22 cases. Crit Care Med. 2000, 28:377-382. 10.1097/00003246-200002000-00015 